\title{
Geant4-DICOM Interface-based Monte Carlo Simulation to Assess Dose Distributions inside the Human Body during X-Ray Irradiation
}

\author{
Sang-Tae Kim \\ Dept. of Radiological Science, Hanlyo Univ., Gwangyang, Jeonnam, Korea
}

\begin{abstract}
This study uses digital imaging and communications in medicine (DICOM) files acquired after CT scan to obtain the absorbed dose distribution inside the body by using the patient's actual anatomical data; uses geometry and tracking (Geant) 4 as a way to obtain the accurate absorbed dose distribution inside the body. This method is easier to establish the radioprotection plan through estimating the absorbed dose distribution inside the body compared to the evaluation of absorbed dose using thermo-luminescence dosimeter (TLD) with inferior reliability and accuracy because many variables act on result values with respect to the evaluation of the patient's absorbed dose distribution in diagnostic imaging and the evaluation of absorbed dose using phantom; can contribute to improving reliability accuracy and reproducibility; it makes significance in that it can implement the actual patient's absorbed dose distribution, not just mere estimation using mathematical phantom or humanoid phantom. When comparing the absorbed dose in polymethly methacrylate (PMMA) phantom measured in metal oxide semiconductor field effect transistor (MOSFET) dosimeter for verification of Geant4 and the result of Geant4 simulation, there was $0.46 \pm 4.69 \%\left(15 \times 15 \mathrm{~cm}^{2}\right)$, and $-0.75 \pm 5.19 \%\left(20 \times 20 \mathrm{~cm}^{2}\right)$ difference according to the depth. This study, through the simulation by means of Geant4, suggests a new way to calculate the actual dose of radiation exposure of patients through DICOM interface.
\end{abstract}

Keywords: DICOM, CT, Fluoroscopy, Geant4, Absorbed dose distribution,

\section{INTRODUCTION}

With concern and anxiety continuing to rise after the disaster at Fukushima Nuclear Power Plant No. 1 on March 13, 2011, it is critical for subjects to have accurate information about their exposure doses during an X-ray test since it can help them reduce their anxiety and allow radiologists or doctors to raise awareness of lower exposure doses.

In particular, it is very important to establish an accurate system for assessing patient exposure doses during an X-ray fluoroscopy, which requires relatively longer radiation exposure time. Essential to the establishment of a more accurate patient doses assessment system is information about dose distributions by considering interactions between the major organs of the patient exposed to radiation and the radiation. Also needed to assess effective doses by multiplying each organ dose by their corresponding tissue weighting factors and adding them up is information about the dose distributions of each organ. It is difficult to directly measure the equivalent doses of various organs when the body is exposed to radiation, which is why humanoid and mathematical phantoms have been used in most patient exposure doses assessments[1]-[3].

The approach, however, should consider some differences between those phantoms and actual human bodies in terms of

\footnotetext{
* Corresponding author, Email: saint-kst@hanmail.net Manuscript received Apr. 01, 2012; revised Jun. 05, 2012; accepted Jun 07, 2012
}

anatomic structure and makes it impossible to assess the radiation exposure doses of actual patients. Thus this study employed DICOM (Digital Imaging and Communications in Medicine) files created after CT scanning using the anatomic information of actual patients to get dose distributions inside their bodies during an X-ray fluoroscopy, as well as Geant (GEometry ANd Tracking)4 instead of EGS (Electron Gamma Shower)4, which has usually been used in radiation therapy, to get accurate dose distributions absorbed in the body. It is estimated that Geant4, which allows the user to pursue functionality, modularity, expandability, and openness in programming, will serve more useful purposes for patient exposure dose assessment and radiation protection plans not only in the domain of high-energy radiation therapy but also low-energy radiation-based diagnosis compared with EGS4, which is comprised of Fortran with $\mathrm{C}++$, an object-oriented language, as its programming language.

One can contribute to greater easiness, reliability, accuracy, and reproducibility by estimating specific dose distributions inside the body and setting medical radiation protection plans rather than conducting dose assessment with TLD (Thermo-Luminescence Dosimeter), which lacks reliability and accuracy due to many variables affecting result values in radiology, a field of radiation-based diagnosis, or human body model phantoms, which have limitations with concretely reproducing anatomic structures including the complicated internal organs. 


\section{MATERIALS AND METHODS}

\subsection{Polymethly Methacrylate (PMMA) Phantom}

The phantom used to measure absorbed doses during CT scanning or fluoroscopy in order to compare with the dose distribution result values of DICOM files-interfaced Geant4 simulation was made of PMMA cubes of $20 \times 20 \times 20 \mathrm{~cm}^{3}$

\subsection{X-Ray Generator}

Brilliance 64 MDCT (Phillips, USA) was used to scan the PMMA phantoms and humans in the abdomen and pelvis. Fluoroscopy was conducted with the under-tube type Easy Diagnost Eleva Inverter R/F (Phillips, USA). All the conditions of X-ray fluoroscopy were adjusted manually.

\subsection{Exposure Conditions}

Table 1 shows the fluoroscopy conditions of a PMMA phantom.

Table 1. The fluoroscopy conditions of a PMMA phantom

\begin{tabular}{|c|c|}
\hline \multicolumn{2}{|c|}{ Fluorography } \\
\hline $\mathrm{SID}(\mathrm{cm})$ & 70 \\
\hline $\mathrm{kVp}$ & 85 \\
\hline $\mathrm{mA}$ & 3 \\
\hline Exposure time $(\mathrm{sec})$ & 150 \\
\hline FOV $\left(\mathrm{cm}^{2}\right)$ & $15 \times 15,20 \times 20$ \\
\hline
\end{tabular}

\subsection{Measurement of Absorbed Doses in a PMMA Phantom}

The MOSFET detector was inserted right in the middle of each PMMA slice (2 $\mathrm{cm}$ thickness) of a PMMA phantom to measure absorbed doses at the depth of 2, 4, 6, 8, $10 \ldots 20 \mathrm{~cm}$ from the bottom of the fluoroscope stand. The dose measurer was calibrated before measurement.

NERO mAx (Model 8000) by Victoreen was used as the ionization chamber to calibrate the output doses of the fluoroscope. The volume and measurement scope of the ionization chamber were $45 \mathrm{cc}$ and $1 \mathrm{mR} \sim 9999 \mathrm{mR}$, respectively. The ionization chamber was also calibrated based on the standard beam quality by Food and Drug Administration before experiments. All the measured doses were adjusted with calibration coefficient of 0.808 [4], which was calibrated with ISO NS 80 with low uncertainty, of the calibration coefficients of high-resolution (RE) low spectrum (NS, resolution range: 27 37). The half-value layer of the fluoroscope was $2.7 \mathrm{~mm} \mathrm{Al}$ at $100 \mathrm{kVp}$ [5]. First, the MOSFET dosimeter was calibrated with the old X-ray tube. For calibration, the MOSFET dosimeter was placed in parallel with the ionization chamber and exposed to $100 \mathrm{kVp} \mathrm{X}$-ray to measure exposure doses. As shown in Fig. 1, the mobile MOSFET dosimeter (TN-RD-70W, Best Medical Canada) was placed in the middle of the phantom to measure doses in the PMMA phantom. Dose measurements were completed right after fluoroscopy, which means those measurements would pass as real-time conditions.

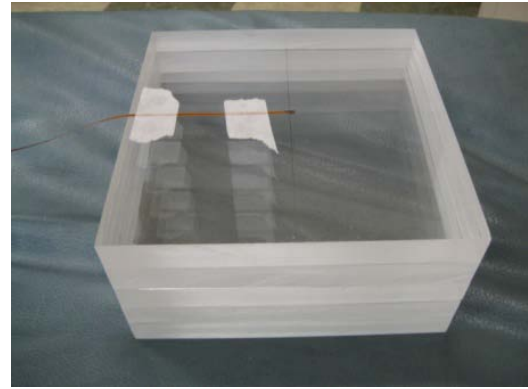

Fig. 1. Insertion of the MOSFET detector in the PMMA phantom

\section{MONTE CARLO SIMULATION}

\subsection{X-ray Spectrum Generation}

The low-energy domain of the X-ray spectrum influences patient exposure doses instead of playing a role in fluoroscopy image creation, which is why the low-energy domain of the $\mathrm{X}$ ray spectrum is eliminated before use by placing an aluminum (Al) filter or $\mathrm{Al}$ and copper $(\mathrm{Cu})$ filter inside the tube.

The X-ray spectrum used for fluoroscopy was set with the technical information of the fluoroscope used in the study and IPEM-78[6]. A code to offer the emitted X-ray spectrum, IPEM-78 is constructed based on the characteristics of the concerned X-ray equipment based on a pool of data including three target matters, namely tungsten, rhodium, molybdenum, the tube voltage range of $30 \sim 150 \mathrm{kVp}$, the target angle of $6 \sim 22^{\circ}$, and filter matters such as aluminum, beryllium, copper, air, and bone. Outputs provide the number of protons per mAs and $\mathrm{mm}^{2}$ (Photons $/ \mathrm{mAs} \cdot \mathrm{mm}^{2}$ ) in energy intervals of $0.5 \mathrm{keV}$. Table 2 contains the $\mathrm{X}$-ray generation conditions of the fluoroscope used in the study. Fig. 2 shows the spectrum generation results of IPEM-78 based on Table 2.

Table 2. X-ray generation conditions of the fluoroscope to generate an X-ray spectrum.

\begin{tabular}{|c|c|}
\hline Target material & Tungsten \\
\hline Tube voltage $(\mathrm{kVp})$ & 85 \\
\hline Anode angle (degree) & 12 \\
\hline Filter & $2.7 \mathrm{~mm} \mathrm{Al}$ \\
\hline
\end{tabular}

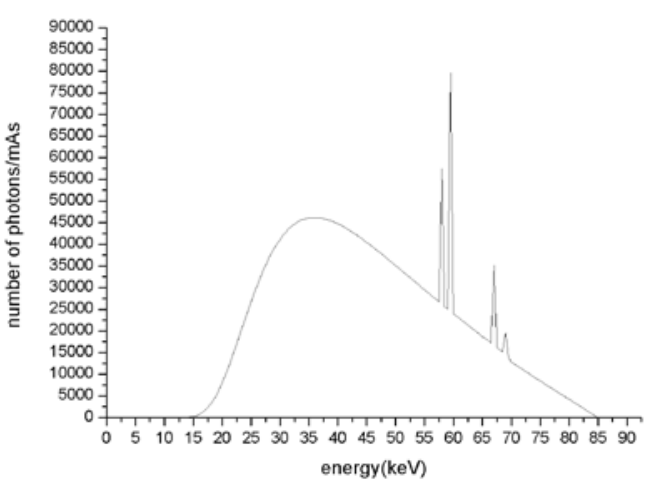

Fig. 2. X-ray spectrum generation 


\subsection{Modeling}

Geant4.9.3p01 was used for modeling in the study. Point sources were assumed and approximated to a fan shape at an angle proper for each radiation field (Table 3 ). Sources were placed at a position $50 \mathrm{~cm}$ distant from the isocenters of the abdomen and pelvis CT DICOM files of the PMMA phantom and actual patient. The fluoroscope stand is mainly comprised of carbon similar to the CT bed, thus absorbing little X-ray. In a simulation designed to calculate dose distributions inside the body, the fluoroscope stand was replaced as in Fig. 3. Then a model of geometric structure similar to that of the under-tube type fluoroscope stand was built to penetrate the CT bed and human body (Fig. 4). Although it is true that X-ray gets scattered in such structures as the image intensifier (I.I. tube) after penetrating the human body and thus contributes to patient exposure doses, such backscattering doses are extremely small compared to primary ray-caused doses. Thus structures X-ray reaches after penetrating the human body such as the image intensifier were not included in the model. The beam histories used in the Monte Carlo simulation were $5 \times 10^{8}, 8 \times 10^{8}$, and $2 \times 10^{9}$ for each radiation field. Adopted in the study was the standard physics model, a physical transportation model for photons and electrons[7,8]. The set cut to determine the secondary particle production threshold value was $0.1 \mathrm{~mm}$ for photons, electrons, and positrons.

Table 3. X-ray beam angle from the point source according to radiation field sizes $(\mathrm{SID}=70 \mathrm{~cm})$.

\begin{tabular}{|c|c|c|}
\hline $\begin{array}{c}\text { X ray field size } \\
(\mathrm{cm})\end{array}$ & $\begin{array}{c}\text { Maxtheta } \\
\text { (degree) }\end{array}$ & $\begin{array}{c}\text { beam angle } \\
\text { (degree) }\end{array}$ \\
\hline 15 & 6.12 & 12.24 \\
\hline 20 & 8.14 & 16.28 \\
\hline 30 & 12.08 & 24.16 \\
\hline
\end{tabular}

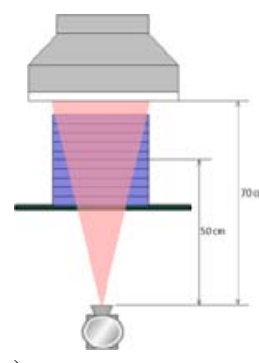

(a)

Fig. 3. Basic structure of the simulation; (a)PMMAphantom (b) Human body

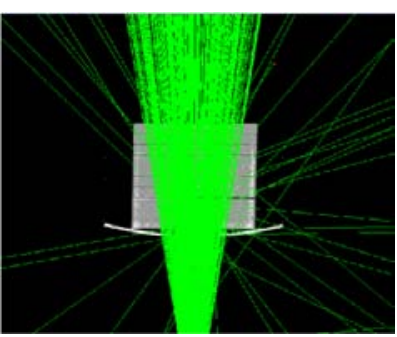

(a)

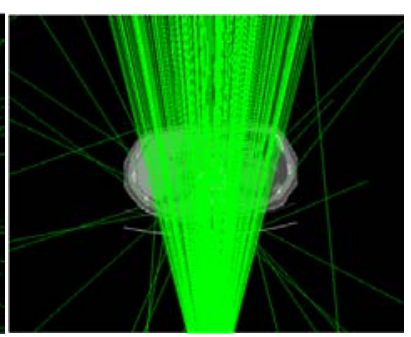

(b)
Fig. 4. X-ray irradiation during simulation; (a)PMMAphantom (b) Human body

\subsection{DICOM Interface}

CT data should be imported into Geant4 to geometrize. DICOM interface was redesigned through partial revision and supplementation at the Geant4 example code to do a simulation proper for the study. DICOM interface converts CT data into such formats as are proper for creating geometry in simulation. CT numbers were categorized into ten representative matters inside tissues in order to reduce memory consumption caused by substance conversion in Geant4 (Table 4). Patients' physical information, which is the subject of simulation, is obtained from CT DICOM files. The interface of DICOM dataset is essential for the purpose.

Although DICOM is a standardized data format, there are differences in the DICOM header formats of dataset among different CT scanner manufacturers, which is why it is critical for Monte Carlo simulation codes such as Geant4 to support various kinds of DICOM dataset. DICOM interface retrieves information from DICOM to build Geant4 geometry. Information used in a geometry model can be classified as follows: DICOM images with CT numbers, pixel intervals, pixel numbers, locations of reference pixels, and image byte orders. There is a table to convert CT numbers into density for the information defined by the user and another one to correspond the cubic cell sizes used in Geant4 geometry construction and molecular structures among organs to CT numbers(Table 4). The area of dose distribution calculation in simulation is important for determining calculation amounts and memory consumption. The sensitive areas where dose distributions are calculated can serve to obtain the patient geometry established by DICOM dataset easily and increase in proportion to calculated amounts and memory consumption.

The substance information in the DICOM files is the CT number, being obtained by converting the attenuation coefficient of radiation detected in the measurer. There should be a conversion process to use the slice information, pixel locations, and pixel Hounsfield information of each ASCIIencoded DICOM file in Geant4 codes. The results will take a shape like in Fig. 5 as the geometric data of Geant4 do not usually match patient data.

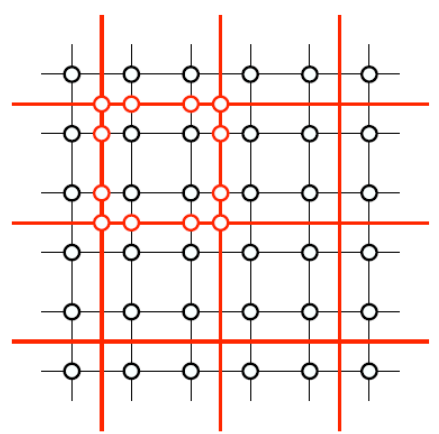

Fig. 5. Relations between DICOM images and Geant4 geometry (Red line : Geant4 geometry border, Black line : DICOM pixel border)

In the figure, the dark solid line represents the voxel data of the patients expressed in the DICOM format, and the grey dotted line the geometric data of Geant 4 . While the dark dots 
represent the Hounsfield values of the DICOM format, the grey dots do the Hounsfield values of Geant4 geometric data and are used in a density conversion process. Linear interpolation is used to interpolate the Hounsfield values, which are the eight neighboring grid points of DICOM pixels, into Geant4 geometric data[9]. Linear interpolation involves drawing a straight line between two points and interpolating according to the ratio. It becomes bilinear interpolation on a two-dimension domain and cubic convolution interpolation on a threedimension domain. Interpolation is carried out for the $\mathrm{x}$ axis.

The result is then interpolated for the $y$ axis, and then there is another round for the $\mathrm{z}$ axis[10]. The Hounsfield value at the location where the points of DICOM image and those of Geant4-converted voxel are extrapolated is obtained through numerical integration.

Density mapping corresponding to the Hounsfield value is one of the important indexes on which the success or failure of precision dose assessment depends in radiation dose distribution assessment. Numerical doses are calculated based on the electron density value to convert into the voxel form of 2 $\mathrm{mm}^{3}$ and distinguish matters through density changes. Table 4 contains the set points in the CIRS Model 062 phantom to correct the CT number and tissue electron density[11,12].

Table. 4. Correlations between CT numbers and certain tissues in the CIRS model 062.

\begin{tabular}{|c|c|c|c|}
\hline Materials & $\begin{array}{c}\text { Physical } \\
\text { density } \\
\left(\mathrm{g} / \mathrm{cm}^{3}\right)\end{array}$ & $\begin{array}{c}\text { relative electron } \\
\text { density } \\
\left(\text { Per } \mathrm{cc} \times 10^{23}\right)\end{array}$ & $\begin{array}{c}\text { CT } \\
\text { number } \\
(\mathrm{HU})\end{array}$ \\
\hline air & 0.001 & 0.001 & 1 \\
\hline lung(inhale) & 0.2 & 0.19 & 230 \\
\hline lung(exhale) & 0.5 & 0.489 & 500 \\
\hline $\begin{array}{c}\text { adipose } \\
\text { tissue }\end{array}$ & 0.96 & 0.949 & 974 \\
\hline breast & 0.99 & 0.976 & 1009 \\
\hline water & 1 & 1 & 1021 \\
\hline muscle & 1.06 & 1.043 & 1061 \\
\hline $\begin{array}{c}\text { liver } \\
\text { trabecular } \\
\text { bone }\end{array}$ & 1.07 & 1.052 & 1074 \\
\hline dense bone & 1.53 & 1.456 & 1146 \\
\hline *HU : Hounsfield Unit & & 1.117 & 1505 \\
\hline
\end{tabular}

Although those density values vary according to correction phantoms and accordingly cannot be regarded as general values, the variations within the range are not big. In the study using Geant4, electron density was replaced with elements for each matter in Table 4. Thus matter density was determined by physical density values. Criteria for corresponding CT numbers to physical density values were based on the values set for Monte Carlo simulations in the previous studies (Fig. 6)[13]. Eight positions were picked, and straight lines were drawn based on their values to determine density corresponding to the overall CT number.

CT numbers were corresponded to physical density values based on the data applied in Schneider and the BEAMnrc code. Schneider corresponded CT numbers to physical density values with stoichiometric correction based on elements. He then applied the findings to 71 human tissues and set air to -1000 950 , the lung to $-949 \sim-120$, soft tissues to $-119 \sim+120$, and bone tissues to $+121 \sim+1600$. Correspondence between CT number and density was set according to the following conditions:

In the BEAMnrc code, air was set to below -950 , the lung to $-950 \sim-700$, soft tissues to $-700 \sim+125$, and bone to +125 or higher. Correspondence between CT number and density was set by linearizing reference points as follows:

Fig. 6 shows a linearized graph by applying the two conditions above.

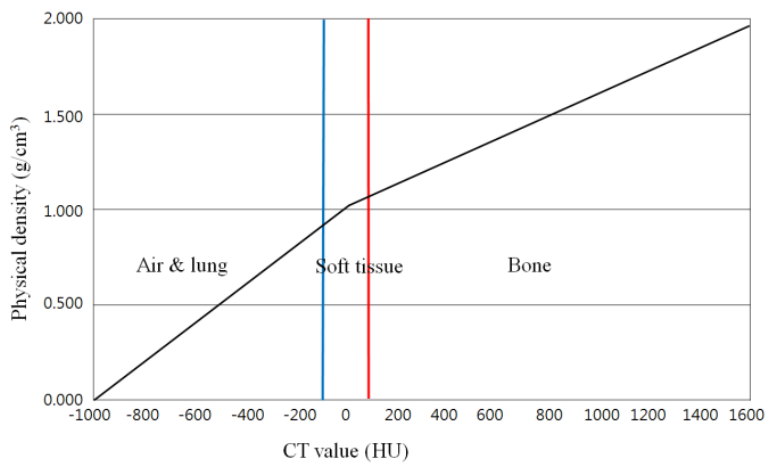

Fig. 6. Linearization for correspondence between CT number and physical density in Monte Carlo simulation

Density calculated in each voxel is a matter accounting for ten tissues. The matter of final voxel is determined through integer transform according to the unique numbers granted to each matter. The recommended density for tissue matters proposed by ICRU Report 46 was used as the density of ten tissue matters. In Fig. 7, (a) is the visualization of DICOM files in JPEG and (b) is DICOM files transformed into g4dcm for the geometry of Geant4. Those files were turned into voxels, and colors were randomly appointed according to density in the videos, which were implemented with the openGL graphics program, one of Geant4 simulation visualization program.

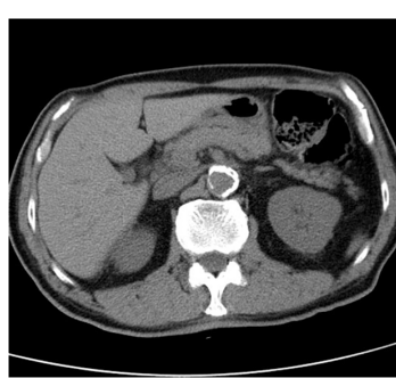

(a)

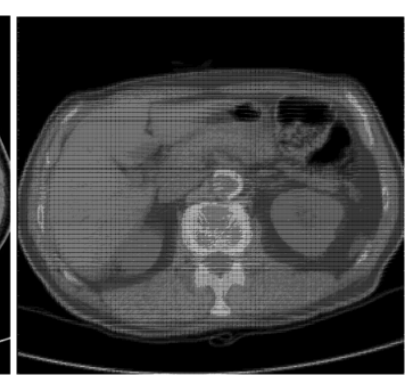

(b)
Fig. 7. Transformed images for geometry at Geant4; (a)

Original DICOM image (b) Transformed image at Geant4

\subsection{Geant4 Dose Normalized Factors (NF)}

Normalized factors were obtained to correct Geant4 simulation calculation values and MOSFET measurement values. Jarry et al.[14] was consulted to obtain measurement 
values for each X-ray radiation field and factors to convert simulation results into doses. MOSFET was placed in the center of radiation field on the PMMA phantom surface $40 \mathrm{~cm}$ distant from the source and corrected to radiation fields of $15 \times 15 \mathrm{~cm}^{2}$ and $20 \times 20 \mathrm{~cm}^{2}$ at $3 \mathrm{~mA}, 150 \mathrm{sec}(450 \mathrm{mAs})$ in order to get absorbed doses by considering backscattering in the same situations as actual dose measurement.

$N F_{\text {energy }}=\frac{\left(D_{\text {mass-measured }}\right)_{\text {energy }}}{\left(D_{\text {mass-sumulated }}\right)_{\text {energy }}}$

$D_{\text {mass-measured }}$ is the measurement of the center of the $15 \times 15$ $\mathrm{cm}^{2}$ and $20 \times 20 \mathrm{~cm}^{2}$ radiation field at $450 \mathrm{mAs}$ with MOSFET. $\mathrm{D}_{\text {mass-simulated }}$ is the simulated calculation value in the same condition. $\mathrm{D}_{\text {mass-measured }}$ was obtained as a dose value per mAs by dividing the measurement value with $450 \mathrm{mAs}$ in order to obtain a normalized factor (NF) for $1 \mathrm{mAs}$. Thus total amounts of $\mathrm{mAs}$ during $\mathrm{D}_{\text {mass-simulated }}$ irradiation are needed to obtain dose values inside the PMMA phantom or human body with a normalized factor.

$D_{\text {absolute }}=N F_{E} \times\left(D_{\text {mass-simulated }}\right)_{E} \times($ Total mAs $)$

Here, E refer to energy and X-ray radiation field, respectively. Table 5 presents normalized factors obtained in such ways.

Table 5. Normalized factors for doses correction when calculating absorbed doses in Geant4 simulation

\begin{tabular}{|c|c|c|c|c|}
\hline $\begin{array}{c}\text { Beam } \\
\text { energy } \\
(\mathrm{kVp})\end{array}$ & $\begin{array}{c}\mathrm{X} \text { ray } \\
\text { field } \\
\text { size } \\
\left(\mathrm{cm}^{2}\right)\end{array}$ & $\begin{array}{c}\text { Measur } \\
\text {-ement } \\
(\mathrm{mGy})\end{array}$ & $\begin{array}{c}\text { Geant4 } \\
\left(10^{-15} \mathrm{mGy} / \text { particle }\right)\end{array}$ & $\begin{array}{c}\text { Normalizati- } \\
\text { on factor } \\
\left(10^{16} \text { particle }\right. \\
/ \mathrm{mAs})\end{array}$ \\
\hline 85 & $15 \times 15$ & 40.22 & $2.63 \pm 0.013$ & 1.52 \\
\hline 85 & $20 \times 20$ & 40.45 & $3.82 \pm 0.011$ & 1.04 \\
\hline 85 & $30 \times 30$ & 41.01 & $4.83 \pm 0.016$ & 0.84 \\
\hline
\end{tabular}

\section{RESULTS}

In the DICOM files that scanned the PMMA phantom, the $\mathrm{x} \times \mathrm{y}$ axis was $284 \times 284 \mathrm{~mm}^{2}$ and the slice interval was $1.5 \mathrm{~mm}$. In the abdomen-pelvis CT DICOM files, the $\mathrm{x} \times \mathrm{y}$ axis was $278 \times 278 \mathrm{~mm}^{2}$ and slice interval was $4 \mathrm{~mm}$. The number of voxels in one slice was $256 \times 256$ when simulating those files. In other words, the voxel size was approximately $1.11 \times 1.1 \times 1.5$ $\mathrm{mm}^{3}$ for the PMMA phantom and for $1.09 \times 1.09 \times 4 \mathrm{~mm}^{3}$ abdomen-pelvis CT. The simulation results were outputted in dose values in all the voxels of a slice.

\subsection{Verification of Geant4 Calculation Codes by Comparing Dosimetry Results}

In case of Monte Carlo calculations with Geant4, the more beam histories are implemented, the less uncertain calculated values become. In the study, however, Monte Carlo calculations were done to obtain absorbed doses at PMMA in the $15 \times 15 \mathrm{~cm}^{2}$ and $20 \times 20 \mathrm{~cm}^{2}$ radiation field; $5 \times 10^{8}$ and $9 \times 10^{8}$ beam histories were implemented in the $15 \times 15 \mathrm{~cm}^{2}$ and $20 \times 20$ $\mathrm{cm}^{2}$ radiation field, respectively. Absorbed doses were obtained at the central axis of each radiation field according to depth at the calculated value of each voxel of DICOM.

Fig. 8 and 9 present MOSFET measurements' comparison with dose distributions in each radiation field assessed through Geant4 simulation and absorbed doses inside the PMMA phantom. MOSFET measurements were higher at most measuring points even though depth doses were very trivial. On the other hand, Geant 4 simulation results were higher at the 6 , 14 , and $16 \mathrm{~cm}$ point of the PMMA phantom from the bottom of the fluoroscope stand. One can possibly take into account errors inevitably made by the MOSFET detector that is not accurately fixed at the points where PMMA slab compression is not complete, does not face the source in the front, and is distorted in direction. However, such differences are small enough to ignore when comparing actual measurements with simulation results. One can also take into account X-ray spectrum transformation before penetrating the body since the fluoroscope stand is flat while the CT bed has some irregularities on the surface despite their similar components. As a result, there can be small differences between Geant4 simulation results and MOSFET dose measurements at certain depths of the PMMA phantom.

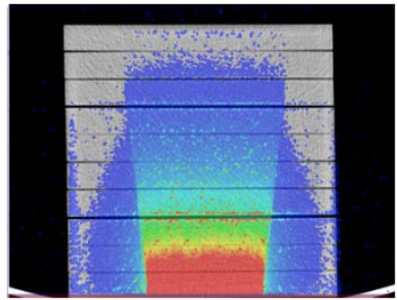

(a)

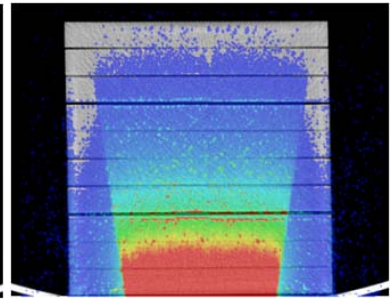

(b)
Fig. 8. Dose distributions inside the PMMA phantom according to changes to the $\mathrm{X}$-ray radiation field size; (a) $\mathrm{X}$-ray radiation field size of $15 \times 15 \mathrm{~cm}^{2}$ (b) X-ray radiation field size of $20 \times 20$ $\mathrm{cm}^{2}$

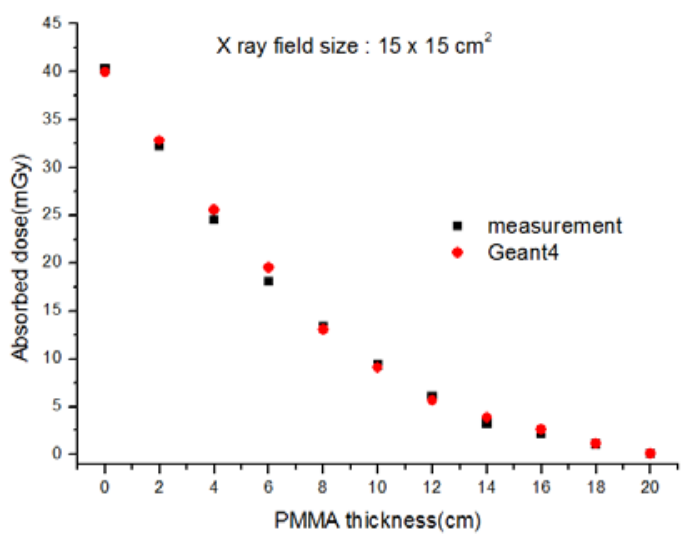

(a) 


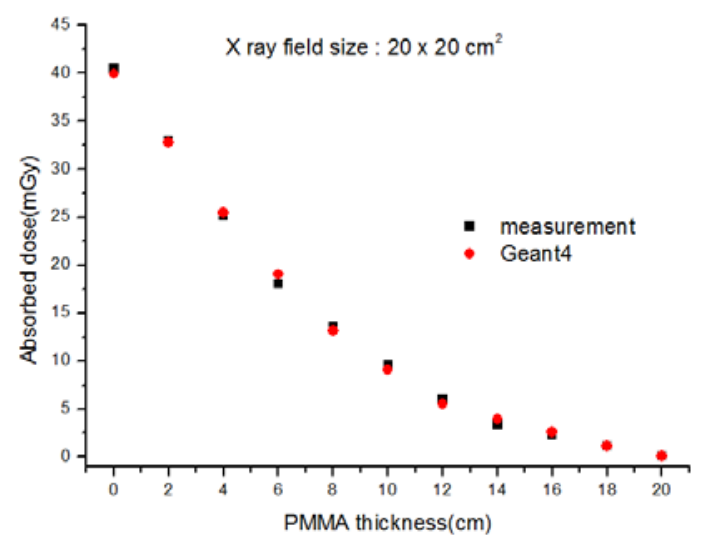

(b)

Fig. 9. between MOSFET measurements and Geant4 simulation results in each radiation field.; (a) Absorbed doses according to PMMA thickness in the X-ray radiation field of $15 \times 15 \mathrm{~cm}^{2}$ (b) Absorbed doses according to PMMA thickness in the X-ray radiation field of $20 \times 20 \mathrm{~cm}^{2}$

\subsection{Abdomen-Pelvis CT DICOM-Interfaced Geant4 Simulation}

The study assessed differences in dose distribution according to the internal organs with the under-tube type fluoroscope, which is commonly used for fluoroscopy, through the interface of Geant4 and CT DICOM. Within the fluoroscopy range, the internal organs recorded 0.035 82.54 mGy of absorbed dose distribution with the pelvis and spine recording relatively higher at $82.54 \mathrm{mGy}$ and $59.88 \mathrm{mGy}$, respectively. Absorbed doses increased in the organs inside the abdomen as the X-ray radiation field grew in size along with the number of organs included in the radiation field. As seen in Fig. 10 and 11, the pelvis recorded the most absorbed doses in the X-ray radiation field of $20 \times 20 \mathrm{~cm}^{2}$, being followed by the spine, small intestine, large intestine, kidney, bladder, stomach, pancreas, spleen, liver, heart, and the lower lobe of the lung in the order. Such dose distributions can be explained by the fact that the X-ray source is irradiated from the back of the subject. The high level of absorbed doses in the spine and pelvis can be explained by high physical density. In the study, the organs within the radiation field during fluoroscopy included the pelvis, spine, kidney, small intestine, large intestine, stomach, liver, pancreas, spleen, and bladder while those outside the radiation field included the lower lobe of the lung, heart, and testicles.

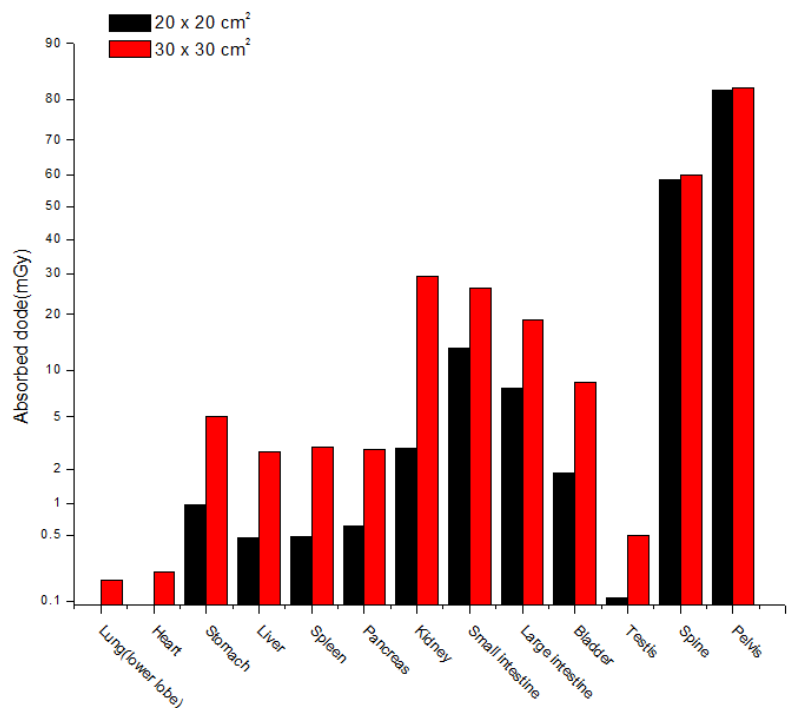

Fig. 10. Absorbed doses in the organs according to radiation field size

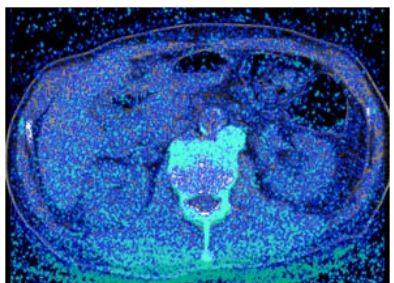

(a1)

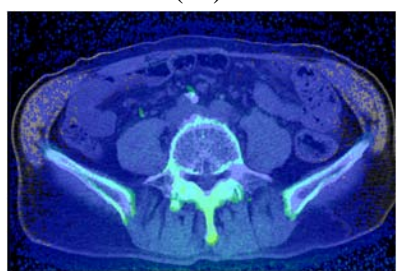

(b1)

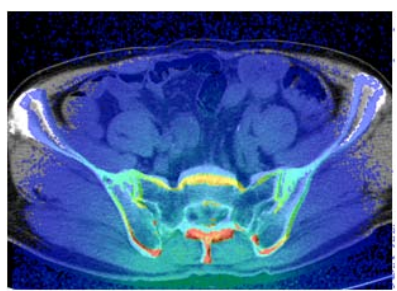

(c1)

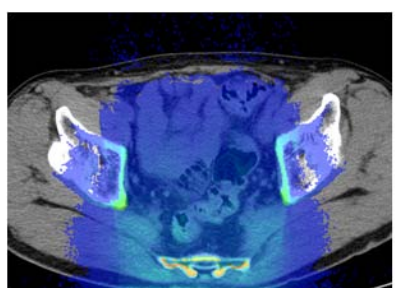

(d1)

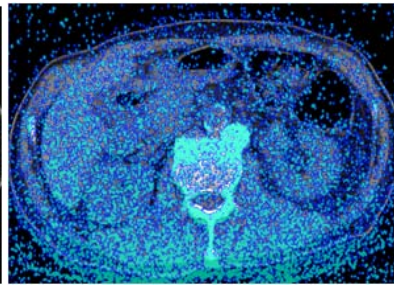

(a2)

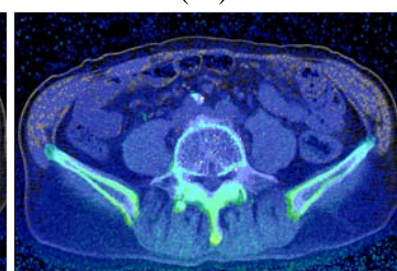

(b2)

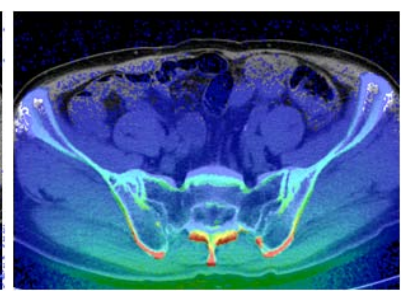

(c2)

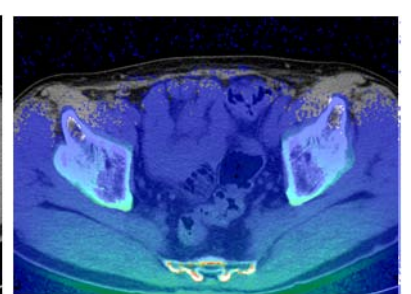

(d2) 


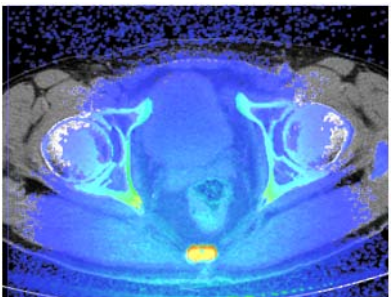

(e1)

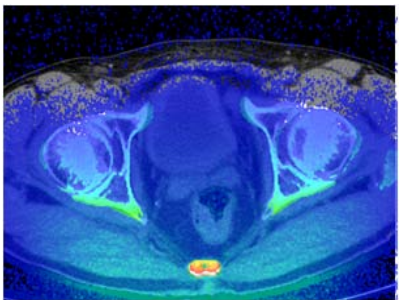

(e2)
Fig. 11. Absorbed dose distributions inside the human body according to radiation field size; (a1) (e1): $20 \times 20 \mathrm{~cm}^{2}$, (a2) (e2) : $30 \times 30 \mathrm{~cm}^{2}$

\section{DISCUSSION}

In the study, dose distribution results inside the body were obtained by comparing Geant 4 simulation results with actual measurements of absorbed doses at the center according to depth in the PMMA phantom with MOSFET dosimetry and interfacing actual patient CT DICOM files reflecting the anatomic information inside the body in order to assess radiation dose distributions inside the body during fluoroscopy. The study thus proposed a new method of calculating radiation exposure doses in actual patients by interfacing DICOM files in Geant4 codes-based Monte Carlo simulation.

In the study, however, the heel effects were not considered when entering photons into PMMA and abdomen-pelvis, which means that one should consider the intensity of incident photons according to cathode and anode in the X-ray tube. Dose distributions will change a little in form by considering it. As for Monte Carlo simulation results, difference between absorbed doses inside the PMMA phantom measured with MOSFET dosimetry and Geant 4 calculation results was up to $9.78 \%$ and $9.69 \%$ in the $15 \times 15 \mathrm{~cm}^{2}$ and $20 \times 20 \mathrm{~cm}^{2} \mathrm{X}$-ray radiation field, respectively. However, those errors were the results of the MOSFET detector being inserted in the PMMA phantom in an unstable direction. It was $0.46 \pm 4.69 \%$ and $0.75 \pm 5.19 \%$ in the $15 \times 15 \mathrm{~cm}^{2}$ and $20 \times 20 \mathrm{~cm}^{2} \mathrm{X}$-ray radiation field, respectively, at all depth points except for those with such errors. It is thus highlighted that the accurate insertion and fixation of the MOSFET detector in the PMMA phantom is important, indeed. As seen with the results, the calculation results of Monte Carlo simulation through interface between Geant4 and DICOM files well matched except for the errors along the border where the MOSFET detector was not accurately fixed due to the incomplete compression of the PMMA phantom. It was also reported that the error range of dose distribution according to the method involving a dummy phantom usually used in patient dose assessment was $\pm 10 \%[15]-[17]$.

However, the error range was reported in the high-energy treatment areas in the unit of $\mathrm{MeV}$. The error range increases in uncertainty in low-energy areas of $400 \mathrm{keV}$ or lower. In particular, surplus measurements following increasing photoelectric effects in areas of $100 \mathrm{keV}$ or lower demonstrate that TLD or PLD devices add more pessimistic aspects in terms of accuracy and reproducibility. The study, of course, produced relatively accurate absorbed dose distributions inside the body comprised of heterogeneous tissues. However, the results do not command absolute reliability. Differences remain in dose distributions even though precision is increased with an ongoing dose correction mechanism. That is why researchers continue to study Monte Carlo simulation to guarantee higher dose accuracy.

It is not easy to calculate dose distributions inside the body with statistical reliability by using patient data (DICOM files) within a short time and common PC memory size when doing Geant4 simulation like in the study. One of the main reasons is the tremendous number of voxels making up the patient geometry. Introduced to meet those requirements in medical application were "nested parameterization volumes" with more efficient functions based on Geant4 collaboration after Version 8.1[18].

Even though such Geant4 code improvements are implemented in a consistent manner, the computation time of Monte Carlo simulation increases in proportion to the number of beams. As a result, hundreds of millions of beams or billions of beams should be used to reduce statistical errors. In other words, hundreds of millions of computations or billions of computations are needed, which means Monte Carlo simulation takes dozens of or hundreds of hours. Researchers thus need higher-function PCs or workstations and further clustering technology to connect many PCs with general specification in a row and allow for computation in order to solve such problems. In recent years, the advancements in CPU technology replaced the single core with the dual core and then the dual core with the quad core. It is time to attempt various methods for faster Monte Carlo simulation. There is a need to establish a system of assessing radiation exposure doses in the internal organs during all kind of radiological tests using radiation through interpretative simulation based on Monte Carlo codes such as Geant4.

\section{CONCLUSIONS}

The findings led to the following conclusions:

1) Theoretical values to test measurements on dosimetry can be obtained through Monte Carlo calculation with interface between Geant4 and DICOM.

2) It is unfeasible to insert dosimetry inside the patient's body and do measurements. Instead, one can obtain the absorbed doses of a target organ through interface between Geant 4 and DICOM.

3) While direct measurement with dosimetry only measures a section of the concerned area, Monte Carlo simulation based on interface between Geant4 and DICOM can devise more efficient radiation protection means and policies by obtaining dose distributions inside the body and visualizing the exposure degree inside the body.

4) South Korea has yet to measure and record patient radiation exposure doses, lacking in efforts for the right use of medical radiation compared with the advanced nations. It will be useful to conduct quantitative video assessment when revising the old testing protocol and setting new ones and build a dose management database for each test to manage patient radiation exposure doses. 
5) Through additional researches, Monte Carlo simulation based on interface between Geant4 and DICOM can be used in individual radiation exposure doses management among the practitioners of medical radiology with high potential risks by radiation as well as the subjects.

In short, Monte Carlo simulation based on interface between Geant4 and DICOM is considered as a very useful means to play a critical role in managing medical radiation exposure, which accounts for most artificial radiation exposure of mankind, at an appropriate level and maintaining its optimization.

\section{REFERENCES}

[1] W. S. Snydner, M. R.Ford and G. G. Warner, "Estimates of Specific Absorbed fraction for Photon Sources Uniformly Distributed in Various Organs of a Heterogeneous Phantom," Society of Nuclear Medicine, New York, MIRD Pamphlet No.5, Revised, 1978.

[2] M. Cristy and K. F. Eckerman, Specific Absorbed Fractions of Energy at Various Ages from Internal Photon Sources, 1. Methods, Appendix A, Description of the Mathematical Phantoms, Health and Safety Research Division, ORNL, 1987.

[3] F. Briesmeister Judith, MCNP-A General Monte carlo Code for Neutron and Photon Transport, LA7396-M, 1986.

[4] Korean Standards Association, $\mathrm{X}$ and gamma reference radiation for calibrating dosemeters and doserate meters and for determining their response as a function of photon energy. Part 1. Radiation characteristics and production methods, KSA ISO 4037-1, 2003.

[5] Philips Healthcare Global Information Center Document. http://incenter.medical.philips.com/doclib/enc/fetch/2000 /4504/577242/577261/577263/670329/670330/5162255/ EasyDiagnost_Eleva_with_digital_Radiography_Fluoros copy_room_solutions_to_combine_both_applications_in _one_room_-

_DRF_applications.pdf\%3fnodeid\%3d5162718\%26vernu m\%3d2. 2009.

[6] Catalogue of Diagnostic X-Ray Spectra and other Data, IPEM, 1997.

[7] K. Amako, S. Guatelli, L. Urban et al., "Comparison of Geant4 Electromagntic Physics Models Against the NIST Reference Data,” IEEE, 42(4), 2005, pp.910-918.

[8] E. Poon, F. Verhaegen, "Accuracy of the photon and electron physics in Geant4 for radiotherapy applications," Med. Phys, 32(6), 2005, pp.1696-1711.

[9] A. Kimura, T. Aso, H. Yoshida et al., "DICOM Data Handling for Geant4-Based Medical Physics Application," IEEE, 4, 2004, pp.2124-2127.

[10] A.-M. Kim, S.-W. Kim, J.-W. Song, et al., "Radiation dose plan system based on particle simulation and volume rendering,” Korea Computer Graphics Society, 12(3), 2006, pp.21-26.

[11] B. Cheng, P. Saw, L. Alphonse et al., "Determination of CT-To_Density conversion relationship for image-based Treatment Planning Systems,” Medical Dosimetry, 30(3),
2005, pp.145-148.

[12] Beardmore et al., "Evaluation of MVCT images with skin collimation for electron beam treatment planning,” J Appl Clin Med Phys, 9(3), 2008, pp.43-57.

[13] W. Schneider, T. Bortfeld, W. Schlegel, "Correlation : between CT numbers and tissue parameters needed for Monte carlo simulations of clinical dose distributions," Phys Med Biol, 45, 2000, pp.459-478.

[14] G. Jarry, J. J. DeMarco, U. Beifuss, C. H. Cagnon and M. F. McNitt-Gray, "A Monte carlo-based method to estimate radiation dose from spiral CT :from phantom testing to patient-specific models," Phys Med Biol, 48, 2003, pp.2645-2663.

[15] J. R. Greig, R. W Miller, P. Okunieff, "An approach to dose measurement for total body irradiation,” Int J Radiat Oncol Biol Phys, 36, 1996, pp.463-468.

[16] P. C.Lee, J. M. Sawicka, G. P Glasgow, "Patient dosimetry quality assurance program with a commercial diode system,” Int J Radiat Oncol Biol Phys, 29, 1994, pp.1175-1182.

[17] E. B. Podgorsak, C. Pla, M. Evans, M. Pla, "The influence of phantom size on output factor, peak scatter factor, and percentage depth dose in large-field photon irradiation,” Med Phys, 12, 1985, pp.639-45.

[18] T. Aso, A. Kiura, T. Yamashita, T. Sasaki, Optimization of Patient Geometry Based on CT data in Geant4 for Medical Application. Nuclear Science Symposium Conference Record, pp.2576-2580, 2007.

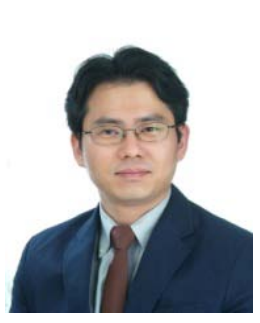

\section{Sang-Tae Kim}

He received the Ph.D. in radiation science and technology from Chonbuk National University, Korea in February 2012. He is currently a professor in the department of radiological science at Hanlyo University. His main research interests include radiation protection and monte carlo simulation. 